\title{
Elaboración de té verde aromatizado con rosas orgánicas "Vitality" de Nevado Ecuador
}

\section{(Development of green tea scented with organic roses "Vitality" from Nevado Ecuador)}

\author{
Elena Beltrán, M. Belén Jácome, Edison Matute
}

\begin{abstract}
Resumen:
El objetivo del presente trabajo fue aromatizar té verde con pétalos de rosas orgánicas "Vitality" de Nevado Ecuador. El té verde, adquirido a una empresa, fue sometido a análisis para verificar el cumplimiento de los requisitos de la norma INEN 2381:2005. Se realizó la caracterización de los pétalos de rosa fresca y se deshidrataron utilizando dos temperaturas y dos geometrías. Se realizaron análisis del contenido de polifenoles totales (método de Folin-Ciocalteu) y capacidad antioxidante (método TEAC). Los pétalos deshidratados, con mayor contenido de polifenoles y capacidad antioxidante, se utilizaron en la elaboración del té verde aromatizado. Tres formulaciones con diferentes contenidos de pétalos de rosa deshidratados (10, 17.5 y $25 \%$ ) fueron analizadas sensorialmente por 100 jueces para determinar el aroma a rosa del té. El producto final fue analizado para determinar el cumplimiento de los requisitos de la norma INEN del té. Finalmente se evaluó la aceptabilidad e intención de compra del producto. Los valores obtenidos de polifenoles totales de los extractos de pétalos de rosa fueron superiores al de frutas como moras, $y$ fresas.
\end{abstract}

Palabras clave: deshidratación; procesamiento de alimentos; tecnología alimentaria.

\begin{abstract}
:
The aim of this study was to obtain a flavored green tea with organic rose petals "Vitality" from Nevado Ecuador. Green tea, purchased from a private company, it was subjected to analysis to verify compliance with the requirements of standard INEN 2381: 2005. The Characterization of fresh rose petals was to made and for the dehydration was used two temperatures and two geometries. Analysis of total polyphenol content (Folin-Ciocalteu) and antioxidant capacity (TEAC method) were performed. The dried petals, with a higher content of polyphenols and antioxidant capacity, were used in the preparation to flavored tea. Three formulations with different amounts of dried rose petals (10,17.5 and $25 \%$ ) were tested sensorially by 100 judges to determine the aroma rose in the tea. The final product was analyzed to determine compliance of the requirements of the standard INEN of the tea. Finally acceptability and purchase intention of the product is evaluated. The values of content total polyphenol in the extracts of rose petals were superior to fruits such as blackberries, and strawberries.
\end{abstract}

Keywords: dehydration; food processing; food technology.

\section{Introducción}

El té es un arbusto de la familia de las teáceas y cuyo nombre científico es Camellia sinensis (Heredia, 2005). Es el producto obtenido de las hojas tiernas, las yemas, brotes, peciolos,

\footnotetext{
${ }^{1}$ Universidad Tecnológica Equinoccial, Facultad de Ciencias de la Ingeniería, Quito - Ecuador (bser2677@ute.edu.ec).
} 
pedúnculos y tallos tiernos y limpios, de las distintas especies del género botánico "Thea” (Gil, 2010), en buen estado de conservación, con el aroma y sabor característicos de su variedad y zona de producción, deshidratados con o sin fermentación (INEN, 2005).

El té aromatizado resulta de la mezcla de té verde, negro u oolong procesado con especias, sustancias aromatizantes autorizadas, hierbas, pétalos de flores, o aceites esenciales de frutas, que le confieren al té un aroma y sabor característico (Heredia, 2005).

Las rosas pertenecen a la familia de las Rosaceae y su nombre científico es Rosa spp. (Sociedad Española de Ciencias Hortícolas, 1998). Para que una rosa sea catalogada como orgánica no debe contener pesticidas y abonos tóxicos, por lo tanto sus cuidados son más rigurosos en comparación con las rosas normales. Para evitar que se dañen y combatir plagas no deseadas se utilizan fertilizantes totalmente naturales e insectos de naturaleza benéfica (Nevado, 2011). La Figura 1 muestra dos de las cuatro variedades de rosas orgánicas cultivadas por la empresa Nevado Ecuador.

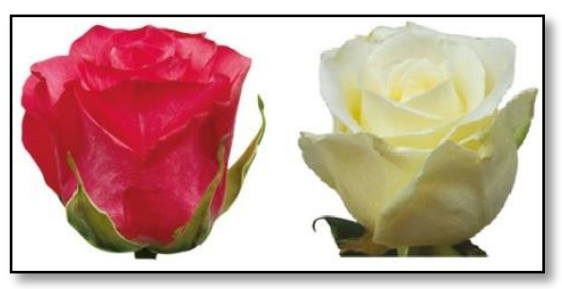

Figura 1. Rosa orgánica roja y blanca (Vitality) de Nevado Ecuador (Nevado Roses, 2012).

La nueva tendencia mundial va encaminada al consumo de pétalos de rosa orgánica, sin embargo para el uso en gastronomía se prefieren aquellas más perfumadas porque su sabor será más marcado (Euroresidentes, 2012; Petryk, 2010). Como todo producto natural, existen algunos beneficios nutricionales asociados con los pétalos de rosas comestibles. Diversos estudios han demostrado que dichos pétalos son una buena fuente de vitamina $\mathrm{C}$ y minerales, especialmente el calcio (Taltavull, 2006).

Uno de los beneficios que los pétalos de la rosa proporcionan al cuerpo humano es la eliminación de toxinas y calor del cuerpo, proporcionándole un cierto efecto refrescante al consumir la infusión. La ingestión de pétalos de la rosa también ayuda a limpiar la garganta así como evitar una nariz mucosa o incluso limpiar los tubos bronquiales bloqueados. También acelera la eliminación de sustancias de desecho a través de los riñones, a la vez que ayuda a combatir enfermedades como la disentería, gastroenteritis y diarrea. Sus características laxantes también lo hacen muy beneficioso tratando problemas como la desnutrición y el estreñimiento. Los pétalos de rosa son también reconocidos porque alivian cualquier síntoma de congestión uterina, que puede causar dolor y contribuye a remediar los conocidos periodos pesados en las mujeres. Por este motivo puede servir como un excelente remedio contra los periodos menstruales irregulares (Mason, 2010). 
Hay rosas de olor suave y delicado, rosas frescas o rosas melosas, rosas afrutadas o rosas muy especiadas, algunas variedades muy especiales tienen matices cítricos muy acentuados. El alcohol 2-feniletanol $\left(\mathrm{C}_{8} \mathrm{H}_{10} \mathrm{O}\right)$ es el principal responsable del aroma típico de las rosas. Se trata de una sustancia muy fragante de olor cálido de rosa con recuerdos de jacinto y es el tipo de sustancia que las personas en general asocian más fácilmente con el olor de la rosa (Botanyuki, 2011).

En el presente estudio se realizó la caracterización de las rosas para conocer su aporte nutricional, así como también el análisis para identificar la presencia de polifenoles y la determinación de la capacidad antioxidante, tanto en rosas frescas como en deshidratadas.

Luego de un proceso de estudio y experimentación, se obtuvieron bolsas de té verde aromatizado con rosas "Vitality" deshidratadas, posibilitando así la formación de un nuevo nicho de mercado para el empleo de dichas rosas en la industria alimentaria.

\section{Materiales y Métodos}

2.1 Caracterización físico química de los pétalos de rosa: Las rosas (Rosa spp.) utilizadas en el estudio provinieron de la florícola "Nevado Ecuador", ubicada en la provincia de Cotopaxi. Las rosas empleadas fueron de la variedad "Vitality", y se diferencian de las otras variedades de rosas orgánicas producidas por la empresa por poseer una fragancia agradable y ser de color blanco.

En la florícola las rosas son tratadas higiénicamente y colocadas en empaques herméticos y sanitizados. Para determinar la composición físico-química de los pétalos de rosa fresca, se realizaron los análisis presentados en la Tabla 1.

Tabla 1. Análisis físico-químicos y metodología utilizados en la caracterización de los pétalos de rosa fresca

\begin{tabular}{|l|l|}
\hline Parámetro & Método \\
\hline Humedad $(\%)$ & NTE INEN 518:81 \\
\hline Proteína $(\%)$ & NTE INEN 519:81 \\
\hline Grasa $(\%)$ & AOAC 960.39 Ed. 18, 2005 \\
\hline Ceniza (\%) & NTE INEN 786:85 \\
\hline Fibra $(\%)$ & INEN 522 \\
\hline Carbohidratos totales $(\%)$ & Cálculo \\
\hline Energía $($ Kcal/100) & Cálculo \\
\hline Sodio $(\mathrm{ppm})$ & Electrodo selectivo \\
\hline Hierro $(\mathrm{mg} / 100 \mathrm{~g})$ & AOAC 944.02 \\
\hline Calcio $(\mathrm{mg} / 100 \mathrm{~g})$ & NOM 185 \\
\hline Colesterol $(\mathrm{mg} / 100 \mathrm{~g})$ & Libermann Burchard \\
\hline
\end{tabular}




\subsection{Deshidratación de los pétalos de rosa}

El proceso de deshidratación de los pétalos de rosa "Vitality" se realizó a dos temperaturas: $35^{\circ} \mathrm{C}$ y $45^{\circ} \mathrm{C}$; y con dos formas geométricas diferentes: pétalos enteros y pétalos cortados en forma de tiras de $1 \mathrm{~cm}$ de ancho, obteniéndose cuatro tratamientos. Se empleó un deshidratado por aire caliente, usando un secador de marca CUISIN PRO, modelo OL 02610 de 600w, equipado con 10 bandejas. Se tomó como peso inicial $150 \mathrm{~g}$.

2.2.1. Contenido de antioxidantes: Se realizó la determinación de polifenoles totales y capacidad antioxidante en los pétalos de rosa antes y después de la deshidratación.

Determinación de polifenoles totales: La determinación del contenido total de polifenoles, se llevó a cabo utilizando el método Folin-Ciocalteu. Se utilizó el método propuesto por Georgé, Brat, Alter y Amiot (2005). Este análisis se lo hizo por triplicado.

Determinación de capacidad antioxidante (abts): La evaluación de la capacidad antioxidante se la realizó mediante el método "Trolox Equivalent Antioxidant Capacity" (TEAC), usando el radical catiónico ABTS. El procedimiento se realizó según el método descrito por Re (1999) y fue realizado por triplicado.

2.3 Análisis sensorial de ordenamiento: Los pétalos deshidratados del tratamiento que presentó un mayor contenido de polifenoles totales y capacidad antioxidante, fueron utilizados para realizar la mezcla con el té verde. Se molieron los pétalos deshidratados con un molino marca BLACK\&DECKER, modelo HC3000. De acuerdo a la norma INEN 2 381:2005, artículo 5.8, al té se le puede adicionar trozos de frutas deshidratadas y/o especies; el porcentaje mínimo de elemento adicionado debe ser del $10 \%$ y el máximo de $25 \%$; para las mezclas se tomaron estos dos extremos y el porcentaje central de $17.5 \%$ y se realizó un análisis sensorial de ordenamiento, en esta prueba participaron 100 personas, se entregó a cada persona tres muestras líquidas de té verde aromatizado con rosas, una por cada formulación. Cada vaso contenía $25 \mathrm{ml}$ de muestra y tenía escrito un código de tres dígitos. Se les pidió que ordenen de forma creciente en cuanto a sensación de aroma y sabor a rosas, para este propósito se entregó a cada persona un cuestionario para cada prueba. Para la interpretación de los resultados se aplicó el análisis de varianza de datos transformados, usando el método de Fisher y Yates, presentado en el libro de Anzaldúa-Morales (1994).

\subsection{Análisis físico químico y microbiológico del té verde y té verde aromatizado}

Se realizó el análisis físico químico del té verde, adquirido a una la empresa privada, y del té verde aromatizado con rosas "Vitality", de acuerdo a la norma INEN 2381:2005 (Té, requisitos). Los análisis y la metodología aplicada se pueden observar en la Tabla 2. 
Tabla 2. Requisitos y metodología aplicada para la realización del análisis físico-químico y microbiológico del té verde y té verde aromatizado.

\begin{tabular}{|l|l|}
\hline Requisito & Método \\
\hline Humedad (\%) & PEE/LA 02 \\
AOAC 925.19
\end{tabular}

\subsection{Etiquetado nutricional y empacado del té aromatizado}

Luego de conocer el resultado del análisis sensorial, se utilizó la formulación con mayor sabor y aroma a rosas y se colocó $1,5 \mathrm{~g}$ de ella en bolsitas de té tradicionales.

Cada paquete de té verde aromatizado con rosas constó de 20 bolsitas, colocados en hilera en cajas de cartón perfectamente cerradas. El exterior del paquete cuenta con indicaciones para la preparación del producto, distintos gráficos que hacen alusión al producto, el peso o contenido neto, el nombre del té y la información nutricional.

La información nutricional del nuevo producto fue realizada, a pesar de que según la norma INEN 1334-2:2011 (Rotulado Nutricional. Requisitos), el té está exento del rotulado nutricional, ya que contienen cantidades insignificantes de todos los nutrientes. Una cantidad insignificante es aquella que permite la declaración de "cero", excepto para carbohidratos totales, fibra alimentaria y proteína cuya cantidad insignificante es menos de un gramo (INEN, 2011).

\subsection{Aceptabilidad del té verde aromatizado}

Se consultó a 100 consumidores habituales de té en distintas cafeterías de la ciudad acerca del producto obtenido en este estudio, donde cada uno probó té aromatizado con rosas "Vitality" y mediante una escala hedónica de 5 puntos, expresaron su grado de satisfacción al probar la muestra. Adicionalmente en esta misma prueba se determinó la intención de compra de los consumidores. 


\subsection{Análisis estadístico}

En la etapa de deshidratación se utilizó un diseño $A x B$, donde las variables dependientes fueron: contenido de polifenoles y capacidad antioxidante (ABTS); mientras que las variables independientes fueron geometría de los pétalos de rosa y temperatura de deshidratación. Los resultados fueron analizados por medio del análisis de varianza y se estableció la comparación de las medias usando el test LSD de Fisher con una significancia de 0,05 empleando el programa Statgraphics Centurion XV.

Para la interpretación de los resultados del análisis sensorial se utilizó el método de análisis de varianza de datos transformados, este método aplica el análisis de varianza, pero transformando antes los datos a valores numéricos según el método de Fisher y Yates, presentado en el libro de Anzaldúa-Morales (1994).

\section{Resultados y Discusión}

\subsection{Caracterización de los pétalos de rosa "Vitality}

Los datos de la Tabla 3 indican los resultados obtenidos del análisis de la composición físicoquímico de los pétalos de rosa fresca de la variedad "Vitality".

Tabla 3. Resultados de los análisis físico-químicos realizados a los pétalos de rosa "Vitality"

\begin{tabular}{|l|c|}
\multicolumn{2}{|c|}{ Paresca } \\
\hline Humedad (\%) & Resultado \\
\hline Proteína (\%) & 85.55 \\
\hline Grasa (\%) & 2.17 \\
\hline Ceniza (\%) & 0.29 \\
\hline Fibra (\%) & 0.74 \\
\hline Carbohidratos totales (\%) & 9.26 \\
\hline Energía (Kcal/100) & 1.99 \\
\hline Sodio (ppm) & 19.25 \\
\hline Hierro (mg/100g) & 2.00 \\
\hline Calcio (mg/100g) & 0.01 \\
\hline Colesterol (mg/100g) & 212.94 \\
\hline
\end{tabular}

En los resultados del análisis de la composición físico-químico de los pétalos de rosa fresca "Vitality" se destaca el alto porcentaje de humedad con $85.55 \%$; el porcentaje de proteína encontrado en los pétalos de rosa fue de $2.7 \%$, este resultado es alto, ya que a modo de comparación, se tiene que vegetales como la lechuga posee un porcentaje de proteínas por debajo del 1.62\% (Pamplona, 2002). Se compara con lechuga por la utilización de pétalos de rosa fresca en ensaladas. 
El contenido de calcio es alto con $212.94 \mathrm{mg} / 100 \mathrm{~g}$, se puede decir que contiene más calcio que la leche de vaca, que posee $120 \mathrm{mg} / 100 \mathrm{~g}$ (Morales, 2007), pero hay que tener en cuenta que esta concentración está sujeta a la biodisponibilidad del calcio en el alimento, por ejemplo el organismo es efectivo absorbiendo el calcio de los lácteos (la biodisponibilidad de estos alimentos es alta) pero no tanto de otros alimentos como las verduras, legumbres, cereales integrales (menor biodisponibilidad), este comportamiento sedebe a que estos alimentos contienen otros componentes que interfieren en la absorción (García \& Aguirre, 2006).

Los pétalos de rosa "Vitality" están constituidos por un $9.26 \%$ de fibra, en comparación con lechuga que contiene 1.5\% (Marín, 2000); su contenido es elevado, pero hay que considerar que la ingesta diaria recomendada de fibra es $35 \mathrm{~g}$, es decir, para satisfacer la necesidad de este nutriente es necesario consumir $378 \mathrm{~g}$ de pétalos de rosa, por lo que para completar la ingesta de fibra se debe comer otros alimentos.

\subsection{Deshidratación de los pétalos de rosa "Vitality"}

Para examinar el comportamiento de los pétalos de rosa durante el proceso de secado, se realizaron las tres curvas típicas de secado de alimentos. En la Figura 2 se puede observar la curva de humedad del producto en función del tiempo.

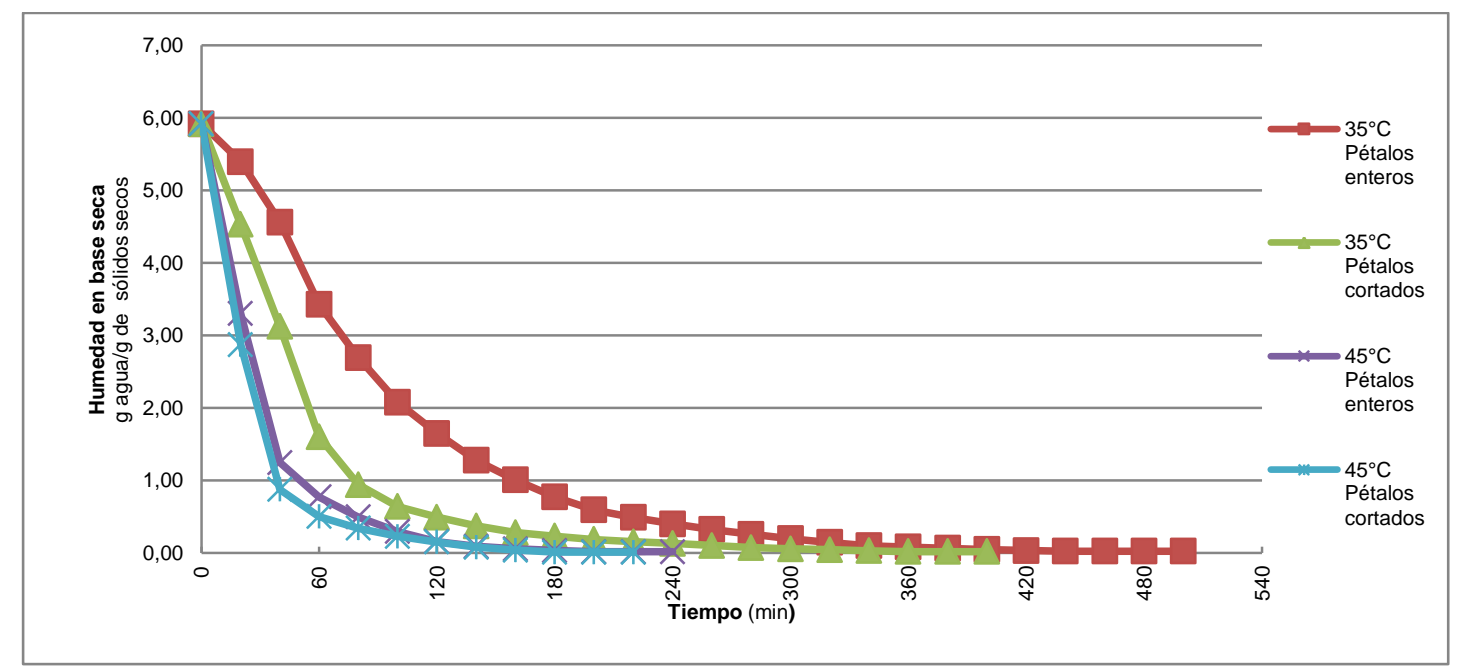

Figura 2. Curva de Humedad vs Tiempo de Pétalos de Rosa "Vitality"

La Figura 2 muestra que a medida que pasa el tiempo la humedad del producto se reduce. La etapa o periodo de estabilización puede ser observado con claridad en el tratamiento de secado de pétalos enteros a $35^{\circ} \mathrm{C}$. En los otros tres tratamientos la etapa de velocidad decreciente empieza inmediatamente iniciado el proceso de deshidratación.

Los procesos de secado a $45^{\circ} \mathrm{C}$ muestran un periodo de deshidratación más rápido que los tratamientos sometidos $35^{\circ} \mathrm{C}$, la diferencia en el tiempo es de casi el doble, siendo el método de pétalos cortados a $45^{\circ} \mathrm{C}$ el que produce un secado más rápido, utilizando 220 minutos; mientras 
que el tratamiento de pétalos enteros a $35^{\circ} \mathrm{C}$ requirió de un mayor tiempo de deshidratación y empleó 500 minutos.

La Figura 3 presenta la curva de velocidad de secado en función del tiempo. La única curva que muestra las cuatro fases del proceso de deshidratación es la del tratamiento de pétalos enteros sometidos a $35^{\circ} \mathrm{C}$, la mayor velocidad de secado se alcanza a los 40 minutos con un valor de 0.0415 (g de agua /g de sólidos secos)/min.

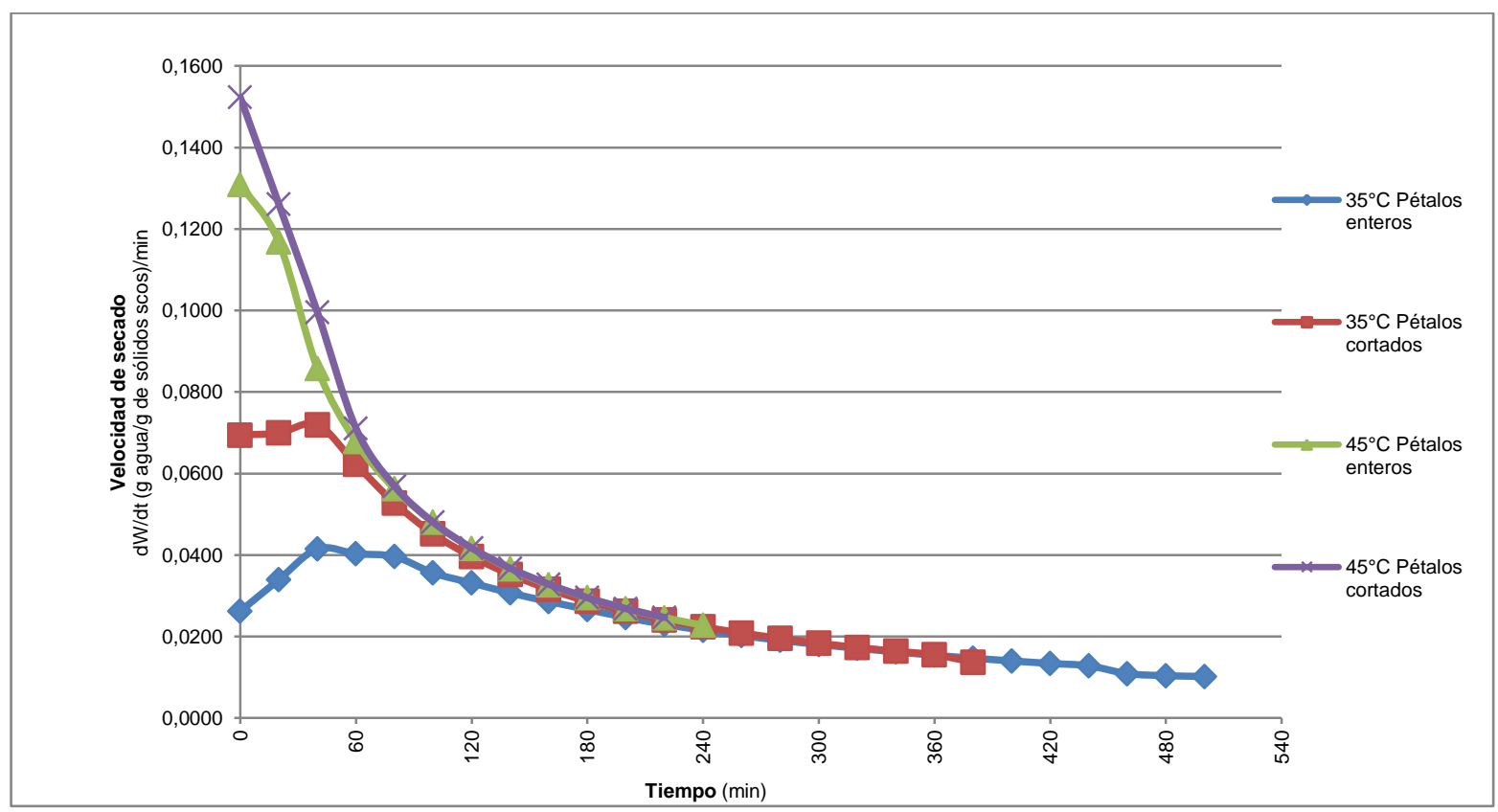

Figura 3. Curva de Velocidad de Secado vs Tiempo de Pétalos de Rosas "Vitality"

El tratamiento de pétalos cortados a $35^{\circ} \mathrm{C}$ también presenta la mayor velocidad de secado en el minuto 40 con 0.072 ( $\mathrm{g}$ de agua /g de sólidos secos)/ min. En los tratamientos que emplean $45^{\circ} \mathrm{C}$, la mayor velocidad de secado se registra al inicio del proceso, luego presentan un descenso de velocidad, y no presentaron periodo de velocidad constante, esto se aprecia con mayor claridad en la curva del tratamiento de pétalos cortados sometidos a $45^{\circ} \mathrm{C}$, que alcanzó su mayor velocidad de secado en los primeros 20 minutos con 1.89 ( $\mathrm{g}$ de agua /g de sólidos secos)/ min.

La Figura 4 representa la curva de velocidad de secado en función de la humedad de los pétalos de rosa.

La ausencia del periodo de velocidad constante se hace evidente al observar las curvas de secado correspondiente a los $45^{\circ} \mathrm{C}$, siendo el periodo de velocidad decreciente el que predominó en todo el proceso; mientras que para los tratamientos que emplearon $35^{\circ} \mathrm{C}$, es posible observar el periodo de estabilización.

La velocidad de secado fue determinada entonces a partir del punto crítico que corresponde a la velocidad máxima de secado, los dos tratamientos sometidos a $45^{\circ} \mathrm{C}$ son las que a mayor 
velocidad de secado llegaron, esto es $0.1309 \mathrm{~g} / \mathrm{min}$ para pétalos enteros y $0.1523 \mathrm{~g} / \mathrm{min}$ para pétalos cortados.

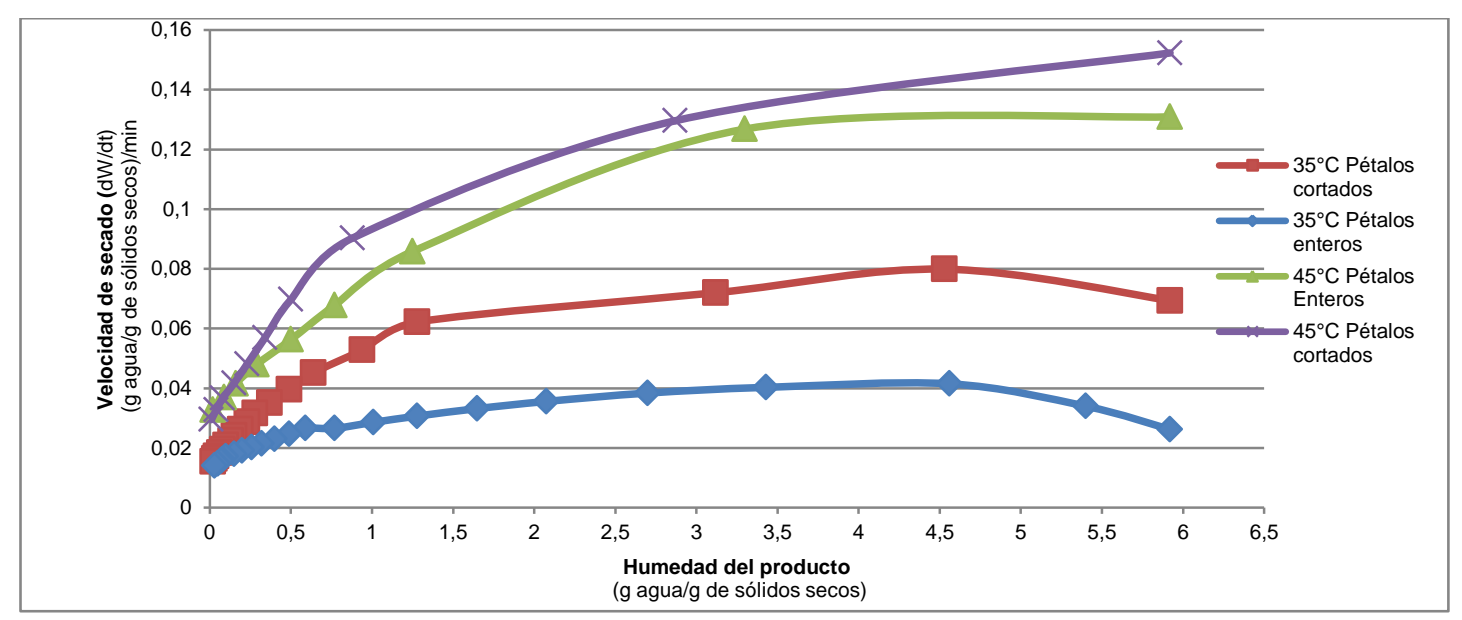

Figura 4. Curva de Velocidad de secado vs Humedad de los Pétalos de Rosa "Vitality"

En la Tabla 4 se presentan de manera resumida los resultados obtenidos de los cuatro tratamientos de deshidratación.

Tabla 4. Resumen de resultados obtenidos después del proceso de deshidratación de pétalos

\begin{tabular}{|l|c|c|c|c|c|}
\hline \multicolumn{1}{|c|}{ Tratamiento } & $\begin{array}{c}\text { Peso } \\
\text { inicial (g) }\end{array}$ & $\begin{array}{c}\text { Peso } \\
\text { final } \\
\mathbf{( g )}\end{array}$ & $\begin{array}{c}\text { Tiempo } \\
\text { (minutos) }\end{array}$ & $\begin{array}{c}\text { Velocidad } \\
\text { máxima } \\
\text { alcanzada } \\
\text { (g/min) }\end{array}$ & $\begin{array}{c}\text { \% Humedad Final } \\
\text { (AOAC 934.06) }\end{array}$ \\
\hline $\begin{array}{l}\mathbf{3 5} 5^{\circ} \text { C Pétalos } \\
\text { enteros }\end{array}$ & 150 & 33 & 500 & 0.04 & 9.92 \\
\hline $\begin{array}{l}\mathbf{3 5}^{\circ} \mathbf{C} \text { Pétalos } \\
\text { picados }\end{array}$ & 150 & 32 & 400 & 0.08 & 9.86 \\
\hline $\begin{array}{l}\mathbf{4 5} \text { C Pétalos } \\
\text { enteros }\end{array}$ & 150 & 31 & 240 & 0.13 & 8.97 \\
\hline $\begin{array}{l}\mathbf{4 5} \text { C Pétalos } \\
\text { picados }\end{array}$ & 150 & 30 & 220 & 0.15 & 8.84 \\
\hline
\end{tabular}

El tratamiento de pétalos cortados sometidos $45^{\circ} \mathrm{C}$ presentó la mayor pérdida de peso. Por otro lado los pétalos que menor tiempo de secado necesitaron fueron los secados a $45^{\circ} \mathrm{C}$, ya que para su deshidratación se empleó 240 y 220 minutos para pétalos enteros y cortados respectivamente.

El tratamiento de $45^{\circ} \mathrm{C}$ con pétalos cortados presentó la mayor velocidad constante, que fue de 0.1523 ( $\mathrm{g}$ de agua /g de sólidos secos)/ $\mathrm{min}$, lo que confirma que la geometría de los pétalos y la temperatura de deshidratación influyen en la velocidad y tiempo de secado.

\subsubsection{Contenido de antioxidantes}

En la Figura 5 se observa que los pétalos de rosa fresca tienen $68.52 \mathrm{mg}$ equivalentes de ácido gálico/g muestra (bs) y presentan el mayor contenido; mientras que el contenido de polifenoles, 
para loa petalos secos, es mayor en los pétalos cortados y en los tratamientos de mayor temperatura, es así que, el tratamiento que presenta el mayor contenido de polifenoles y diferencia significativas con los otros tratamientos $(p<0.05)$ es el de pétalos cortados a $45^{\circ} \mathrm{C}$ con un valor de 57.09 mg equivalentes de ácido gálico/g muestra.

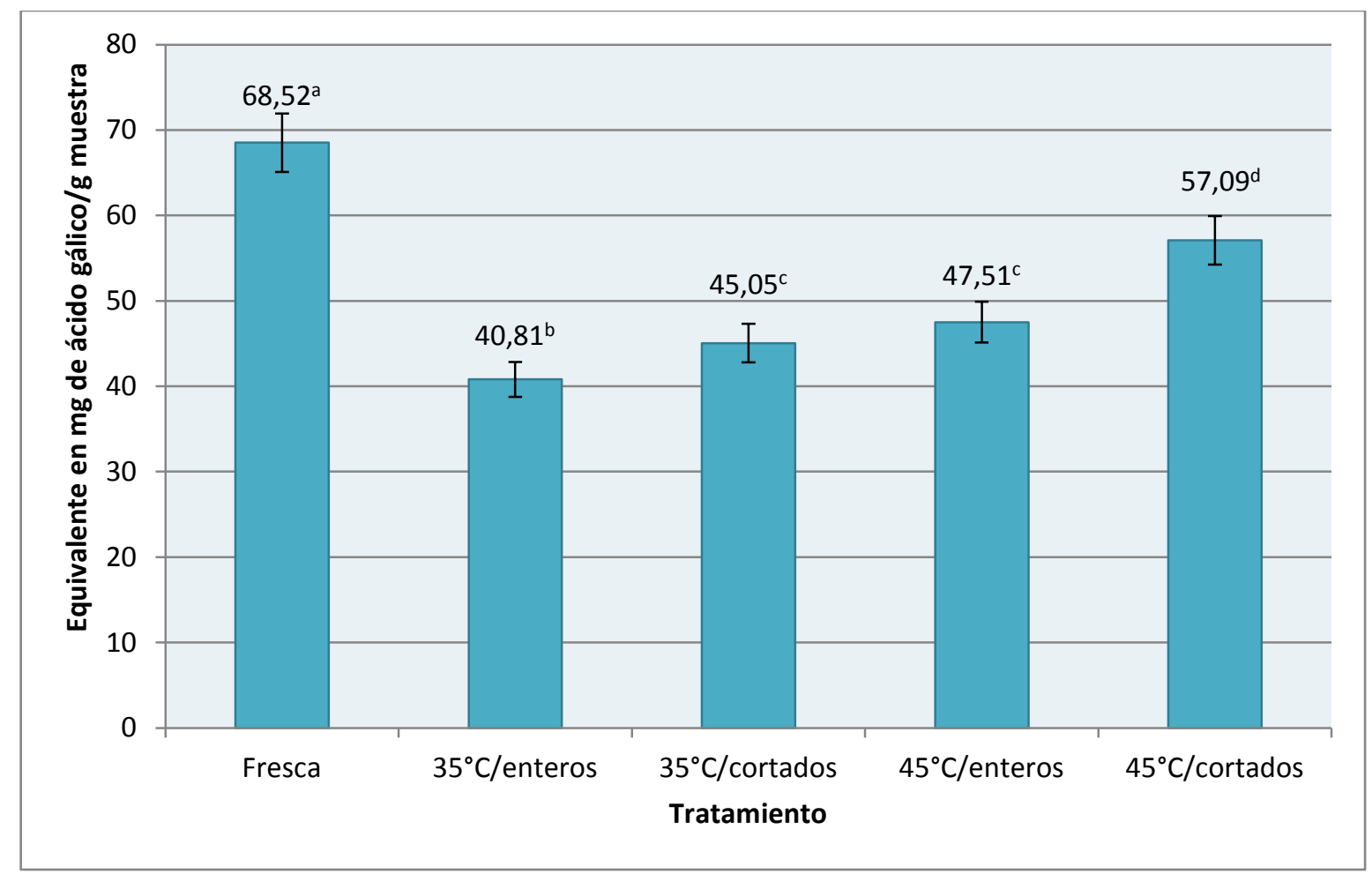

Figura 5. Polifenoles totales (mg de ácido gálico/ gr de muestra, base seca) de pétalos de rosa "Vitality".

Los pétalos de rosa "Vitality" presentaron mayor contenido de polifenoles que algunas frutas, por ejemplo el kiwi tiene la cantidad máxima de polifenoles totales de 30 eq en mg AG/g muestra seca determinado por el método Folin-Ciocalteu (Díaz Flores, 2009).

Los valores obtenidos de polifenoles totales de los extractos de pétalos de rosa "Vitality" (40-57 eq en $\mathrm{mg}$ de AG/g muestra seca) llegan a ser superiores al de frutas como moras, ciruelas, fresas y pomelos tienen un contenido de polifenoles totales de alrededor de 0.3-10; 1.7-3.5; 1.6-2.5 y 1.6 eq en $\mathrm{mg}$ de AG/g peso seco, respectivamente (Bover Millera, 2009). Situación similar se presenta en vegetales como brócoli, col y espinacas, algunos de los vegetales más ricos en fenoles totales, que presentan valores de entre 0.3-3; 0.5-1, y 0.3-1 eq en $\mathrm{mg}$ de AG/g peso seco, respectivamente (Bover Millera, 2009).

La capacidad antioxidante presenta diferencia significativa $(p<0.05)$ entre los tratamientos de secado a $45 \stackrel{\circ}{\circ}$ y la muestra fresca; en Figura 6 se observa que el tratamiento que mostró la mayor capacidad antioxidante fue a $45^{\circ} \mathrm{C}$ con pétalos cortados, con un valor $0.248 \pm 0.009 \mu \mathrm{mol}$ equivalente de Trolox/g muestra y para los pétalos frescos el valor fue de $0.197 \pm 0.010 \mu \mathrm{mol}$ equivalente de Trolox/g muestra. Estos resultados coinciden con la tendencia de los resultados de 
contenido de polifenoles, donde mientras más temperatura se presenta y la superficie de secado es menor, mayor concentración de polifenoles se hace presente y mayor es la capacidad antioxidante, esto significaría que existe una correlación entre el contenido de polifenoles y la capacidad antioxidante.

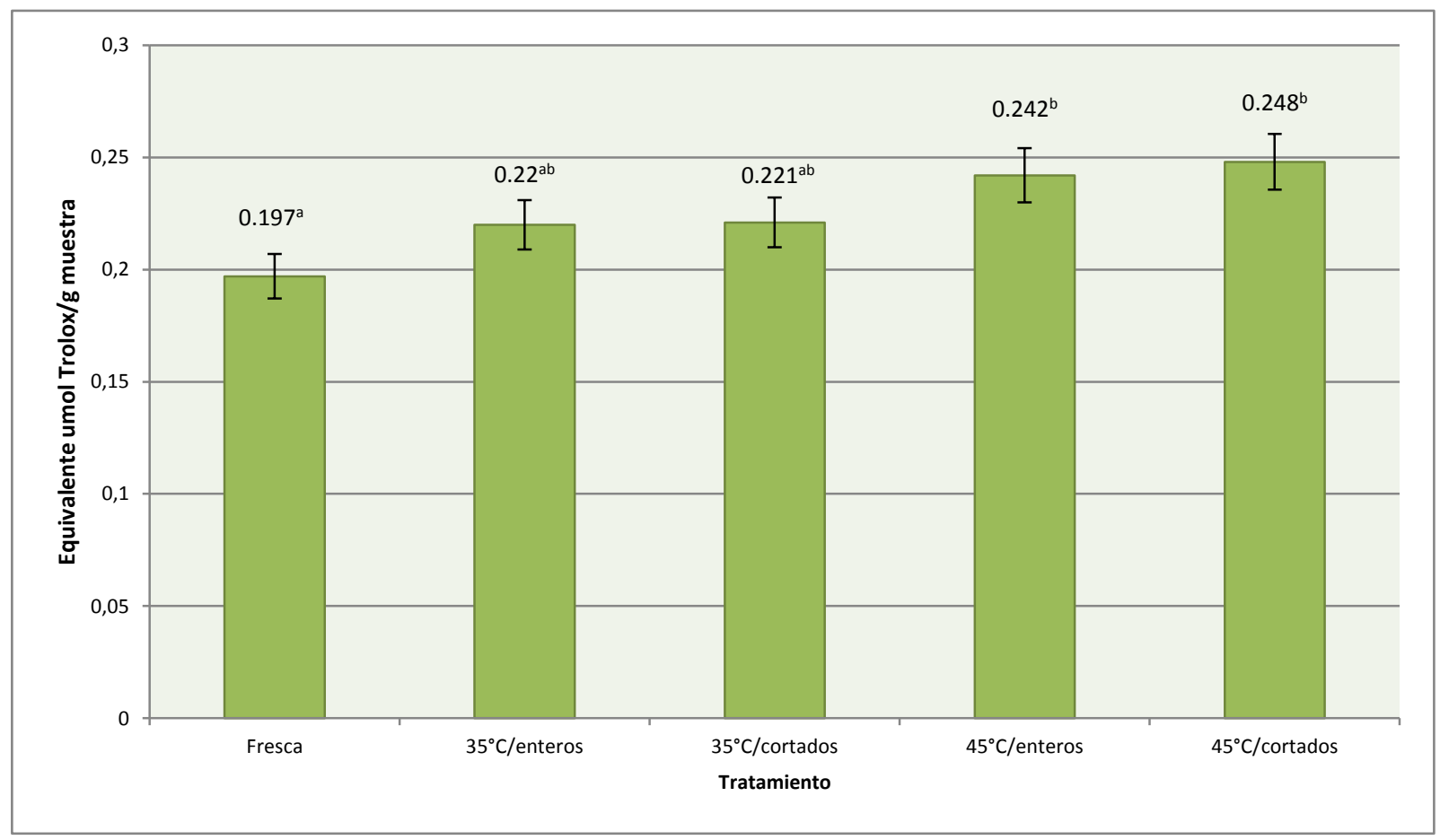

Figura 6. Capacidad antioxidante (Equiv $\mu \mathrm{mol}$ Trolox/g muestra) de pétalos de rosa "Vitality"

Los resultados obtenidos en cuanto a capacidad antioxidante coinciden con estudios realizados por Bover (2009) a pétalos claros de pensamientos, clavel blanco, margarita blanca y amarilla, girasol y lisiantus blanco, que cuentan con valores por debajo de 0.2 meq Trolox/g muestra. El color blanco en las flores se debe, entre otras cosas, a las diminutas bolsas de aire entre las células que lo forman, lo que explicaría la ausencia casi total de compuestos con actividad antioxidante en las flores de este color, especialmente de antocianos (Bover Millera, 2009).

\subsection{Análisis sensorial de ordenamiento}

Los resultados de la prueba de ordenamiento para evaluar aroma y sabor de las tres formulaciones de té verde aromatizado con pétalos de rosa "Vitality", se presentan en la Figura 7.

En ambos atributos (sabor y aroma), el análisis de varianza revela que existe diferencia estadística significativa entre las tres formulaciones, siendo la formulación que contiene $75 \%$ de té verde y $25 \%$ de rosas "Vitality" la que mayor sensación de aroma y sabor a rosas dejó en las personas consultadas. 


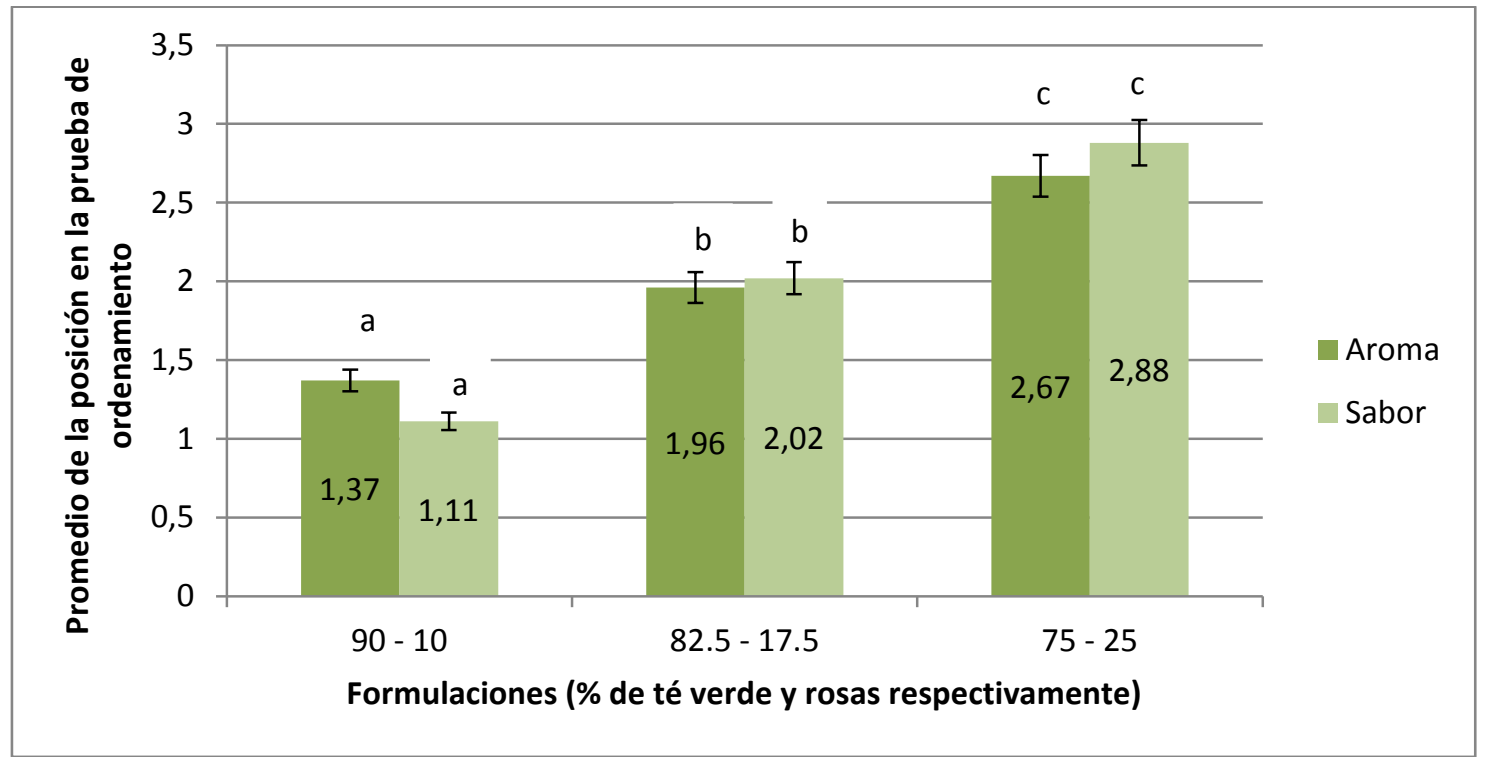

(NOTA: Letras diferentes en las formulaciones de té verde aromatizado con rosas, en cuanto a sabor y aroma, denotan diferencia estadística significativa $(P<0.05)$ y valor de Tukey=0.2147)

Figura 7. Ordenamiento promedio de la sensación de aroma y sabor a rosas de té verde aromatizado con rosas orgánicas "Vitality"

\subsection{Análisis físico químico y microbiológico del té verde y del té verde aromatizado}

Los resultados de los análisis del té verde y té verde aromatizado, así como los límites establecidos por la norma INEN 2381:2005 (Té, Requisitos) se presentan en la Tabla 5.

Tabla 5. Resultados de los análisis físico-químicos y microbiológicos realizados al té verde

\begin{tabular}{|c|c|c|c|c|}
\hline \multirow{2}{*}{ Parámetro } & \multicolumn{2}{|c|}{$\begin{array}{c}\text { Norma INEN } \\
\text { 2381:2005 }\end{array}$} & \multirow{2}{*}{ Té verde } & \multirow{2}{*}{$\begin{array}{l}\text { Té verde } \\
\text { aromatizado }\end{array}$} \\
\hline & Min & Max & & \\
\hline Humedad (\%) & -- & 12 & 6.05 & $7.68 \pm 0.05$ \\
\hline Ceniza (\%) & 4 & 8 & 4.64 & 4.39 \\
\hline Cenizas insolubles en ácido (\%) & -- & 1.0 & 0.97 & 0.97 \\
\hline Cenizas solubles en agua (\%) & 45 & -- & 53.50 & $41.87 \pm 0.67$ \\
\hline $\begin{array}{l}\text { Alcalinidad de las cenizas } \\
\text { solubles en agua (como } \mathrm{KOH}), \%\end{array}$ & 1.0 & 3.0 & 2.93 & $1.1 \pm 0.01$ \\
\hline Plomo (mg/L) & -- & 0.5 & 0.09 & 0.08 \\
\hline Arsénico (mg/L) & -- & 1.0 & 0.1 & 0.05 \\
\hline Cafeína (\%) & 1 & -- & 4.1 & $1.09 \pm 0.01$ \\
\hline \multicolumn{5}{|c|}{ Análisis microbiológicos } \\
\hline Parámetro & \multicolumn{2}{|c|}{$\begin{array}{l}\text { Norma INEN 2381:2005 } \\
\text { Max }\end{array}$} & Té verde & $\begin{array}{l}\text { Té verde } \\
\text { aromatizado }\end{array}$ \\
\hline $\begin{array}{l}\text { Recuento de Aerobios Mesófilos } \\
\text { (ufc/g) }\end{array}$ & \multicolumn{2}{|c|}{$1.0 \times 10^{4}$} & $54 \times 10$ & $37 \times 10$ \\
\hline $\begin{array}{l}\text { Recuento de Coliformes Totales } \\
\text { (ufc/g) }\end{array}$ & \multicolumn{2}{|c|}{$<10$} & $<10$ & $<10$ \\
\hline Recuento de Mohos (upm/g) & \multicolumn{2}{|c|}{$2.0 \times 10^{3}$} & $61 \times 10$ & $13 \times 10$ \\
\hline
\end{tabular}

Los resultados del análisis físico químico del té verde se encuentran dentro de los límites establecidos por la Norma INEN 2381:2005. El análisis microbiológico determinó que el recuento 
de aerobios mesófilos, coliformes y mohos realizados en el té verde, se encuentran dentro de los límites permisibles de la norma y es apto para el consumo.

Los resultados obtenidos del análisis microbiológico del té verde aromatizado con rosas "Vitality", se presentan en la tabla 5 observándose que el producto cumple con los requisitos microbiológicos dispuestos por la norma INEN 2381 referente al té.

El té verde aromatizado con rosas orgánicas "Vitality" presentó un porcentaje de cenizas solubles en agua que se encuentran ligeramente por debajo del mínimo permitido, este resultado se debe a las rosas deshidratadas tienen menor cantidad de estos compuestos y para cumplir con este requisito se debería cambiar el porcentaje de pétalos deshidratados al $24,4 \%$, en lugar del $25 \%$, con lo que el producto podría salir al mercado sin inconvenientes.

\subsection{Análisis nutricional y empacado del té verde aromatizado}

De acuerdo con la norma INEN1334-2:2011, se utilizó como base a una dieta de 8380 kJ (2000 calorías) para obtener la información nutricional del té verde aromatizado con rosas, además se empleó como porción $1.5 \mathrm{~g}$. En la Tabla 6 se observan los resultados del análisis nutricional del té verde aromatizado, esta tabla irá impresa en cada caja de té verde aromatizado con rosas.

Tabla 6. Información nutricional del té verde aromatizado con rosas orgánicas "Vitality"

\begin{tabular}{|l|r|}
\hline \multicolumn{2}{|l|}{$\begin{array}{l}\text { Porción: } 5 \mathrm{~g} \\
\text { Número de porciones: } 20 \\
\text { Cantidad por porción }\end{array}$} \\
\hline Energía 0 kJ (Calorías 0 Cal) & Energía de grasa 0 kJ (Calorías de grasa 0 Cal) \\
\hline \multicolumn{2}{|c|}{$\%$ Valor diario* } \\
\hline Grasa Total 0g & $0 \%$ \\
\hline Grasa Saturada 0g & $0 \%$ \\
\hline Colesterol 0mg & $0 \%$ \\
\hline Sodio Omg 0 & $0 \%$ \\
\hline Carbohidratos totales 0g & $0 \%$ \\
\hline Fibra Dietética 0g & $0 \%$ \\
\hline Azúcares 0g & $0 \%$ \\
\hline Proteína 0g & $0 \%$ \\
\hline
\end{tabular}

* Valores diario requerido en base a una dieta de 8380 kJ (2000 calorías)

El valor encontrado en cuanto a los diferentes nutrientes fue muy bajo e insignificante y de acuerdo a lo establecido por la norma INEN 1334-2, se reportaron con el valor de cero y el producto está exento de los requerimientos de etiquetado nutricional.

La mezcla de té verde y pétalos de rosa "Vitality" deshidratados (75\% y $25 \%$ respectivamente) se envasó en bolsitas de papel filtro con $1.5 \mathrm{~g}$ de la mezcla y se selló junto con un hilo de cáñamo, que contó en el extremo con una etiqueta. Finalmente se colocaron 20 bolsas de té verde aromatizado con rosas "Vitality" en forma de hilera en cajas de cartón, que en su parte exterior 
contienen imágenes que hacen alusión al nuevo producto, la forma de preparación de la infusión además de la información nutricional presentada en la Tabla 6 . En la Figura 8 se puede apreciar el producto empacado
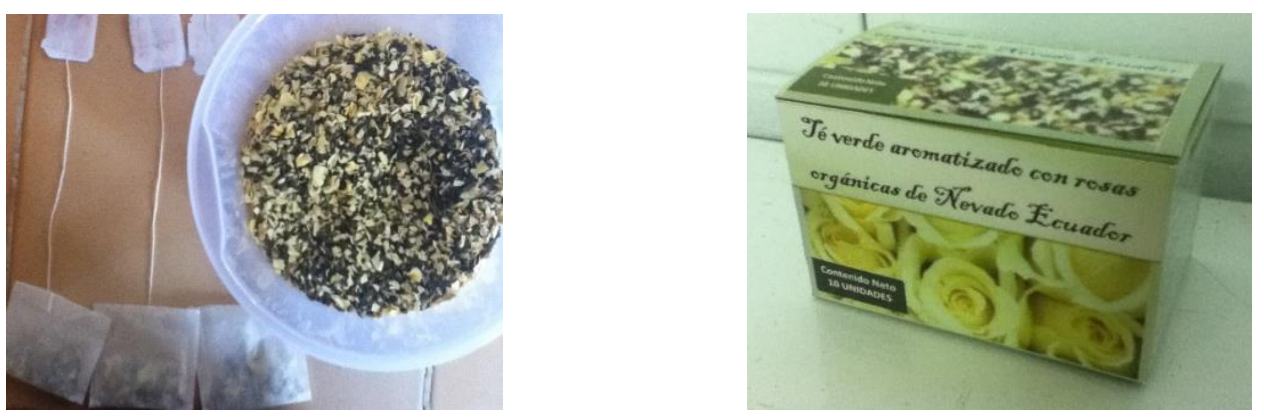

Figura 8. Té verde aromatizado con pétalos de rosa en su empaque para la venta

\subsection{Prueba de aceptabilidad e intención de compra del té verde aromatizado}

El producto final fue evaluado mediante una prueba de aceptabilidad, aplicada a 100 consumidores frecuentes de té, usando una escala hedónica de 5 puntos; los resultados se presentan en la Figura 9.

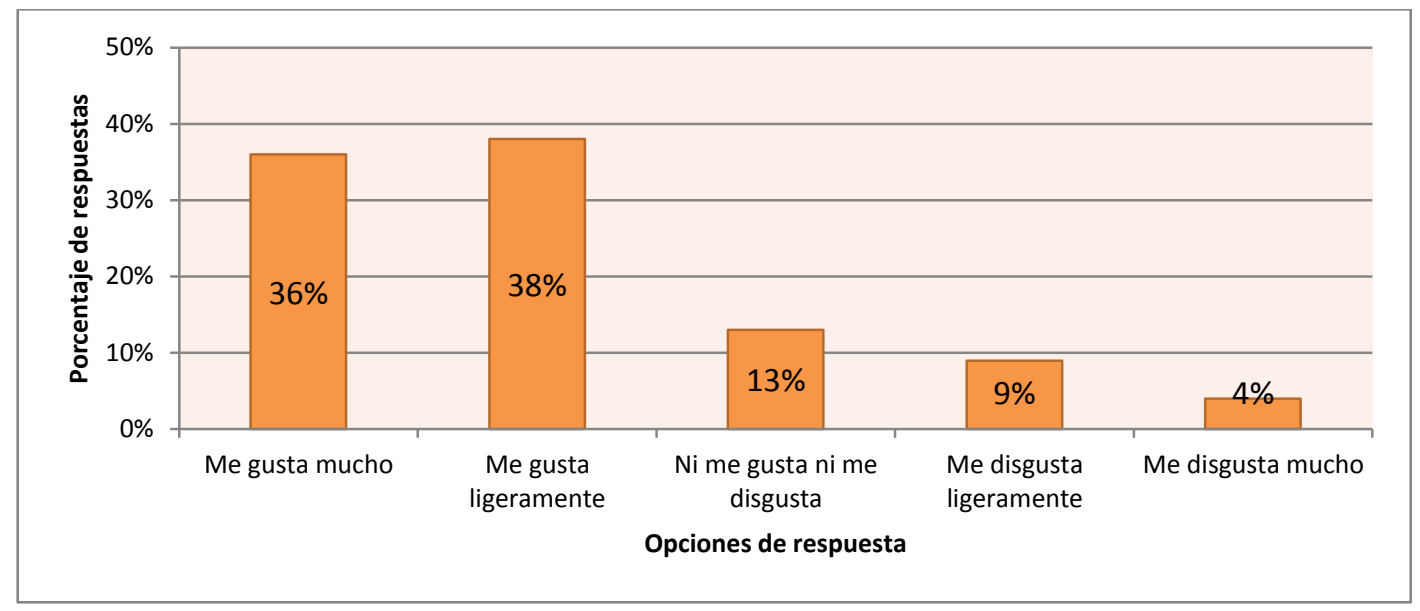

Figura 9. Resultados de la prueba de aceptabilidad del té verde aromatizado con rosas orgánicas "Vitality".

De las 100 personas consultadas, 38 contestaron que les gusta ligeramente el nuevo producto, mientras que un $36 \%$ de los encuestados, respondieron que les "gusta mucho". Un 13\% respondieron que "ni les gusta ni les disgusta"; por otro lado es baja la cantidad de respuestas negativas para producto, ya que 9 personas indicaron que el té verde aromatizado les "disgusta ligeramente" y apenas un $4 \%$ de los encuestados respondieron que el producto les "disgusta mucho". De estos resultados se concluye que el producto tiene una alta aceptabilidad.

En cuanto a la intención de compra, de las 100 personas que participaron en la encuesta, un $82 \%$ respondió que si comprarían el nuevo producto una vez que salga el mercado; mientras que un $18 \%$ respondió negativamente. 


\section{Conclusiones y Recomendaciones}

Se determinó que los pétalos de rosa deshidratados a $45^{\circ} \mathrm{C}$ y cortados en forma de tiras son los que poseen la mayor capacidad antioxidante con 0.248 umol equivalente Trolox/g muestra, de la misma manera presentan una mayor presencia de polifenoles con $57.096 \mathrm{mg}$ de ácido gálico/g muestra, estos parámetros fueron utilizados para escoger este tratamiento para la mezcla del té verde aromatizado.

Los análisis físico químicos y microbiológicos realizados al té verde aromatizado con rosas orgánicas "Vitality", se ajustan a lo que exige la norma INEN 2 381:2005 (Té requisitos), por lo que este producto se constituye una alternativa de innovadora de consumo de rosas en la industria alimentaria.

Se recomienda: experimentar con otras temperaturas y geometrías de los pétalos de rosa "Vitality" para su deshidratación, utilizando secadoras de aire caliente; establecer un estudio para aplicar radiación UV-C a los pétalos de rosa fresca para generar una mayor concentración de antioxidantes; emplear los pétalos de rosa "Vitality" deshidratadas para formular nuevas mezclas con otras variedades de té como el negro, rojo, blanco u oolong.

\section{Bibliografía}

Botanyuki. (14 de Mayo de 2011). Intro al aroma de la rosa. Recuperado el 10 de Septiembre de 2011, de Olibanum World Press: http://olibanum.wordpress.com/2011/05/14/intro-al-aromade-la-rosa-i-rosa-fresca-toda-fragancia/

Bover Millera, L. (2009). Estudio y cuantificación de la actividad antioxidante de flores comestibles. Catalunya: Universidad Politécnica de Catalunya.

Díaz Flores, J. (2009). Optimización de extracción y análisis de la capacidad antioxidante de la piel de kiwi . Catalunya: Universidad Politécnica de Catalunya.

Euroresidentes. (2012). Flores en la cocina. Recuperado el 9 de Junio de 2012, de Euroresidentes: http://www.euroresidentes.com/Alimentos/hierbas/flores-en-la-comida.htm

FAO. (2006). LECHUGA ( Lactuca sativa L.): FAO. Recuperado el 10 de Diciembre de 2012, de FAO:

http://www.fao.org/inpho_archive/content/documents/vlibrary/AE620s/Pfrescos/LECHUGA. HTM

Ferrantelli, P. (2005). Encliclopedia Práctica de la Medicinas Alternativas. Buenos Aires: Ediciones Lea. 
García, C., \& Aguirre, M. (2006). Dieta y calcio para el niño:Traumatología Infantil. Recuperado el 11 de Diciembre de 2012, de Traumatología Infantil: http://www.traumatologiainfantil.com/contenidos/pdf/Dieta\%20calcio.pdf?PHPSESSID=3b0 0f3c3289b2c0db5e1346ded67f2f1

Heredia, M. (2005). Descubra el poder del té. Buenos Aires: Grupo Imaginador de ediciones.

INEN. (2005). Té. Requisitos. Quito: Instituto Ecuatoriano de Normalización.

INEN. (2011). Rotulado de productos alimenticios para consumo humano. Parte 2. Rotulado nutricional. Requisitos. Quito: Instituto Ecuatoriano de Normalización.

La casa del té. (mayo de 2003). La elaboración del té. Recuperado el 10 de Agosto de 2011, de La casa del té: http://www.casadelte.com/laelaboracion.htm

Marín, Z. (2000). Elementos de Nutrición Humana. San José de Costa Rica: Editorial Universidad Estatal a Distancia, San José, Costa Rica.

Mason, S. (18 de Noviembre de 2010). Rose Petals- Edible and good for health. Recuperado el 12 de Septiembre de 2011, de Diet Health Club: http://www.diethealthclub.com/articles/497/diet-and-wellness/rose-petals-edible-and-goodfor-health.html

Melo, V., \& Cuamatzi, O. (2007). Bioquímica de los procesos metabólicos. México: Editorial Reverté.

Morales, J. (2007). Nutriterapia, salud y longevidad. Madrid: Diaz de Santos.

Nevado Roses. (2012). Organic Roses. Recuperado el 25 de Junio de 2012, de Nevado Roses: http://www.nevadoroses.com/roses/rose-varieties/organic-roses/white-roses/rosevarieties/8-organic-roses/or004/P72-white.html

Nevado, R. (23 de Agosto de 2011). Rosas orgánicas. (E. Matute, Entrevistador)

Pamplona, J. (2002). Salud por los alimentos. Madrid: Editorial Safeliz.

Petryk, N. (2010). Nuevas Tendencias:Flores comestibles. Recuperado el 19 de enero de 2012, de Alimentación sana.

Sociedad Española de Ciencias Hortícolas. (1998). Diccionario de Ciencias Hortícolas. Madrid: Mundi-Prensa.

Taltavull, M. (2006). Comer bien y vivir mejor. Buenos Aires: Ediciones LEA. 